\title{
Unstructured road detection based on hybrid features
}

\author{
Erke Shang, Xiangjing An, Lei Ye, Meiping Shi, Hongtao Xue and Hangen He \\ The College of Mechatronics and Automation \\ National University of Defense Technology \\ Changsha, P.R. China \\ Email: erke1984@163.com
}

\begin{abstract}
Unstructured road detection is a key step of the Unmanned Guided Vehicle (UGV) system for road following. In traditional methods, kinds of features are combined to improve the effect, but few of them explains the reason to choose feature descriptors. In this paper, we try to find a way that how to choose feature descriptors. First, support vector machine technology is used to analyze the importance among these common feature descriptors during the road detection process. A series of features are analyzed to show their ability to detect road surface from background. Then, a mass of experiment results verify the correct of our analysis. At last, a novel unstructured road detection algorithm based on hybrid features is proposed. Experiments conducted on actual roads illustrate the effective performance of the proposed algorithm.
\end{abstract}

Keywords: unstructured road detection; feature descriptor; support vector machine

\section{INTRODUCTION}

ROAD detection is a key requirement for the Unmanned Grand Vehicles (UGV). Unstructured road detection is a difficult job due to its unstructured natures. For example, the road edge border may be unclear and have low intensity contrast; the overall road shape may be arbitrary; the road surface can be degraded appearance [1]. On the other hand, varying illumination conditions, different viewpoints or changing weather conditions make the problem more complexity. Fig. 1 shows the same scene in different lighting conditions, weather conditions and viewpoints. In these conditions the road feature extraction is difficult and the detection becomes inaccurate.

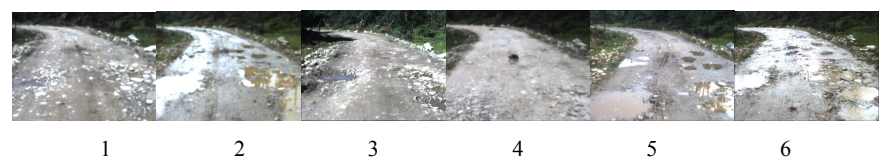

Fig. 1. The same scene in different lighting conditions, weather conditions and viewpoints.

Many algorithms have been proposed to deal with unstructured road detection in last decades. The previous methods can be mainly divided into three groups: model-based method[2], [3], feature-based method[1], [4], and machine learning-based method[5], [6]. Road model has been widely used in many algorithms. They attempt to determine mathematical models to fit the lane boundaries. The choice of the models is of great difficult. A simple model may be robust and easily calculated, but may not provide an accurate result. A complex model may be flexible and suitable for various shapes of road, but may be affected easily by disturbance[7]. The main advantage of the feature-based method is that it is insensitive to the shapes of roads, and it needs only a little previous knowledge. However, it is difficulty to choose a suitable feature descriptor. The machine learning-based method is thought time-cost and it can not meet the real-time requirements[3].

In this paper, we are focus on the feature-based methods. In general, feature-based methods use kinds of features or multi-features for road detection [1], [8], [9], [10], [11]. Paper [1] chooses an advanced RGB values feature to detect road surface, while the average of RGB values is used in [6]. On the other hand, a series of features are combined to improve the effectiveness of road detection in [11], [8]: feature of RGB color models, feature of road distribution models, and its graylevel intensity feature comprising a lane vector are obtained via simple image processing. To the best of the authors' knowledge, no researcher give rules to choose which feature is the most important during the process of unstructured road detection. If there is a method to show the importance of these kinds of feature descriptors, road detection method would be improved greatly. In this paper, we try to use support vectors machine (SVM) technology to analyze the importance of each feature descriptor during the process of road detection. Then, a novel unstructured road detection algorithm is proposed based on hybrid features. Experiments show that the proposed algorithm is effectiveness.

The remainder of this paper is organized as follows. Section II discusses a series of different feature descriptors and analyzed their importance by using SVM technology. Section III describes the experiment result of road detection by using kinds of different feature descriptors. A novel unstructured road detection algorithm corresponding the most important feature and feature combinations is also presented. Experiments show that the proposed algorithm greatly enhances the ability of unstructured road detection, and can exactly detect the road region. Section IV concludes the paper. 


\section{SERIES OF FEATURE DESCRIPTORS IN ROAD DETECTION}

Among feature-based road detection methods, the effects of road detection are greatly depended on kinds of variable feature descriptors. Many texture-based feature descriptors are used in road detection: the average of RGB values, the average of difference RGB values, the histogram of RGB values, the histogram of difference RGB values, the advanced RGB values[1], and so on. To the best of the authors' knowledge, no researcher has to analyze which feature descriptor is the most important during the road detection. In this chapter, we try to analyze the effectiveness of these feature descriptors and find the most important feature descriptor to the unstructured road detection. In order to simplifier the problem, only five feature descriptors mentioned above are analyzed in this paper. It is the same way to analyze other feature descriptors.

\section{A. The average of $R G B$ values}

RGB information is the most widely used feature during the road detection. The average of RGB values is a typical RGB information during a sampling process. The original image is first divided into many small patches. The size of each patch is $n \times m$. The average of RGB represents the average value of each patch $\left\{R_{\text {avg }}, G_{a v g}, B_{a v g}\right\}$.

$$
\left\{\begin{array}{l}
R_{\text {avg }}=\frac{\sum_{i=1}^{n \times m} R_{i}}{n \times m} \\
G_{\text {avg }}=\frac{\sum_{i=1}^{n \times m} G_{i}}{n \times m} \\
B_{\text {avg }}=\frac{\sum_{i=1}^{n \times m} B_{i}}{n \times m}
\end{array}\right.
$$

In our experiments, the patch size is $10 \times 10$. In each patch, the average of color value is divided into 10 pieces. So the vector size of the average of RGB values is $10 \times 3=30$.

\section{$B$. The average of difference $R G B$ values}

In many cases, different color channels in unstructured road surface and background are greatly different. For example, green channel is greater than others in the surface of glass, but is smaller in road surface where is dirt. Thus, the average of difference RGB values R_G,G_B,B_R is also widely used in road detection algorithms. It is defined as follows:

$$
\left\{\begin{array}{l}
R \_G=R_{a v g}-G_{a v g} \\
G \_B=G_{a v g}-B_{a v g} \\
B \_R=B_{a v g}-R_{a v g}
\end{array}\right.
$$

The vector size of the average of difference RGB values in our experiment is $10 \times 3=30$, too.

\section{The histogram of $R G B$ values}

The definition of the histogram of RGB values is similar to histogram of orientation gradient (HOG)[12], they capture local shape information by encoding RGB gradients in histograms. The advantage of this feature descriptor is that different textures in an image will express differently in histogram.

In our experiment, the number of bin in each color channel is 8 , thus the vector size of the RGB histogram is $8 \times 3=24$.

\section{$D$. The histogram of difference $R G B$ values}

The histogram of difference RGB uses $\left\{R \_G, G \_B, B \_R\right\}$ instead of original $\{R, G, B\}$ when computing their histograms. Thus, the vector size of difference RGB histogram is $8 \times 3=$ 24 , too.

\section{E. The advanced $R G B$ values}

Paper [1] presented an advanced RGB feature $\left\{R_{\text {nor }}, G_{\text {nor }}, B_{\text {nor }}\right\}$ to detect road surface. Original RGB values are translated into normal RGB values as follows equations:

$$
\left\{\begin{array}{l}
R^{\prime}=\frac{Y_{\text {avg }}}{R_{\text {avg }}} \times R \\
G^{\prime}=\frac{Y_{\text {avg }}}{G_{\text {avg }}} \times G \\
B^{\prime}=\frac{Y_{\text {avg }}}{B_{\text {avg }}} \times B
\end{array}\right.
$$

and

$$
\left\{\begin{array}{l}
R_{\text {nor }}=\frac{R^{\prime}}{R^{\prime}+G^{\prime}+B^{\prime}} \\
G_{\text {nor }}=\frac{G^{\prime}}{R^{\prime}+G^{\prime}+B^{\prime}} \\
B_{\text {nor }}=\frac{B^{\prime}}{R^{\prime}+G^{\prime}+B^{\prime}}
\end{array}\right.
$$

Where $Y_{a v g}=\left(R_{a v g}+G_{a v g}+B_{a v g}\right) / 3$. In our experiment, the vector size of the advanced RGB values is as the same as the average of RGB values, $10 \times 3=30$.

\section{F. analyzing the effectiveness of these different feature de- scriptors}

The SVM algorithm is chosen to analyze the effectiveness of these feature descriptors. SVM is a technique motivated by statistical learning theory. Its key idea is to separate two classes with an optimal decision hyper-plane that has maximum margin using the training sample. The most important point in feature descriptors would decide the decision hyper-plane. The main idea in this paper is that using the support vector to show the importance of these different feature descriptors during the unstructured road detection. Thus, our experiment is designed as follows:

Step1: a set of positive samples and a set of negative samples are generated from variable road surface and background (Fig.2).

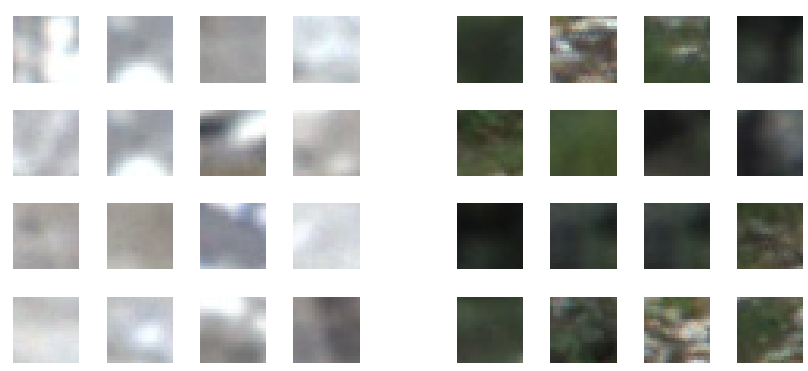

The set of positive samples

The set of negative samples

Fig. 2. The sets of training samples. 
Step2: translate these samples into training data according to different feature descriptors, positive training data $P=$ $\left\{P_{1}, P_{2}, \cdots, P_{n}\right\}$, negative training data $F=\left\{F_{1}, F_{2}, \cdots, F_{n}\right\} . P_{i}$ means that it was produced by $i^{\text {th }}$ feature descriptor.

Step3: trains a SVM classifier using the matrix of training data taken from two groups generated in step2. Information about the trained SVM classifier is returned in a SVM structure.

Step4: observe the support vectors in the SVM structure. The support vectors show the importance of each feature descriptor during the road detection (Fig.3).

Obviously, from Fig.3, we can see that some kinds of features (such as the histogram of RGB values, the advanced RGB values and the average of different RGB values) are more important than other features (such as the histogram of difference RGB values) during the process of unstructured road detection.

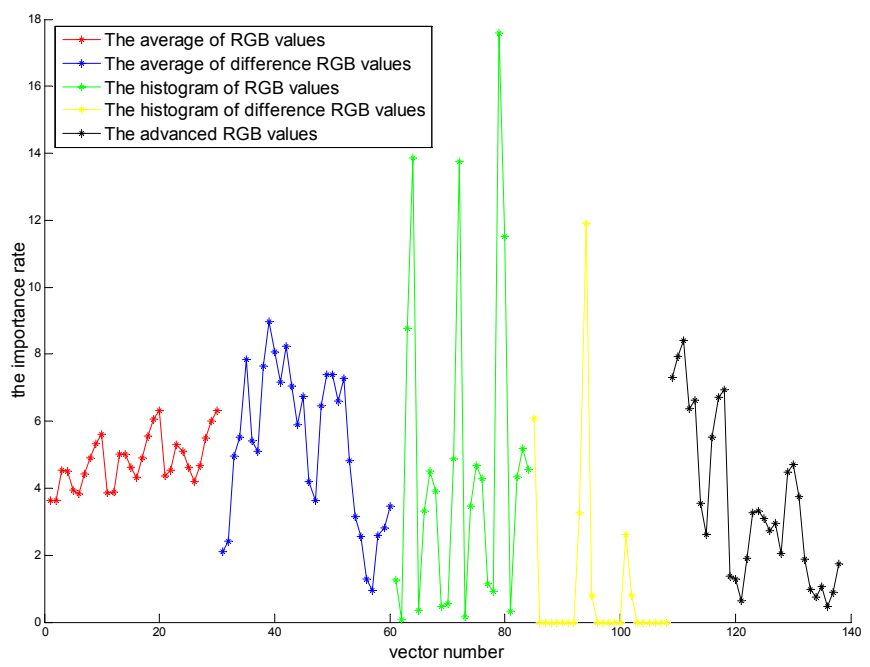

Fig. 3. Support vectors corresponding different feature descriptors.

\section{EXPERIMENTS AND RESULTS}

In order to verify the result analyzed above, actual road experiments are carried out based on these feature descriptors. In order to analyze the importance of different feature descriptors during the unstructured road detection and simplify the problem, kinds of feature descriptors + SVM-based method is chosen. In traditional, the SVM classifier returns property " +1 " means road region or "-1" means background region. We use the margin distance instead of that property (upper part in Fig.4(b)), in which these pixels with deeper color exhibiting higher probability to be road region. The results of road detection based on these five feature descriptors are shown in Fig.4. Fig.4(a) is the benchmark labeled by human. The upper row in Fig.4(b,c,d,e,f) are the deep map of margin distance generated by SVM according different kinds of feature descriptors. The lower row in Fig.4(b,c,d,e,f) are the result of region detection when a fixed threshold is chosen. Different ranges of thresholds are marked besides. Experiment

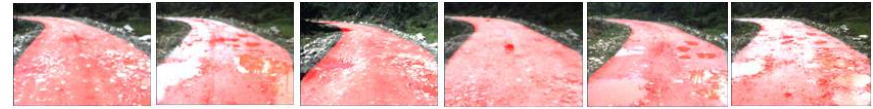

(a) Benchmark (by hand)
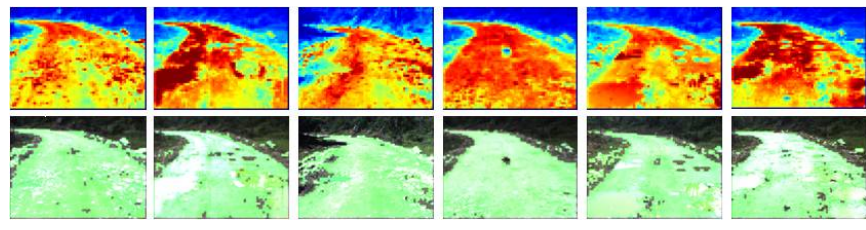

(b) The average of RGB value, threshold $=300$ [200:600]
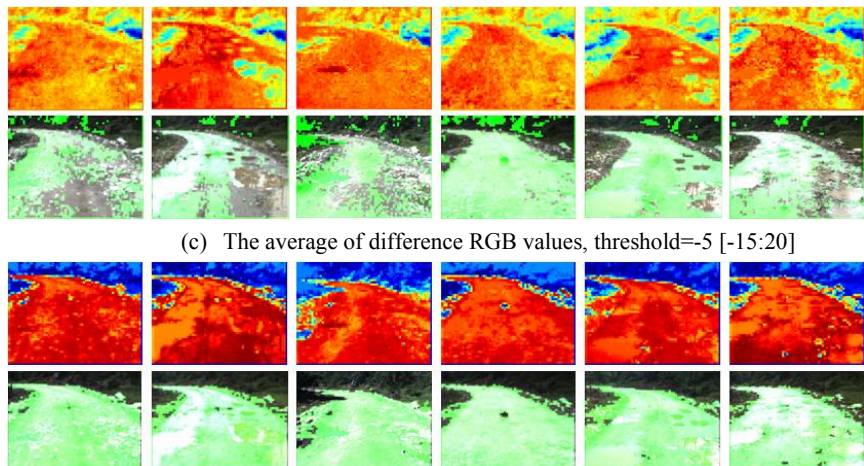

(c) The average of difference RGB values, threshold $=-5$ [-15:20]
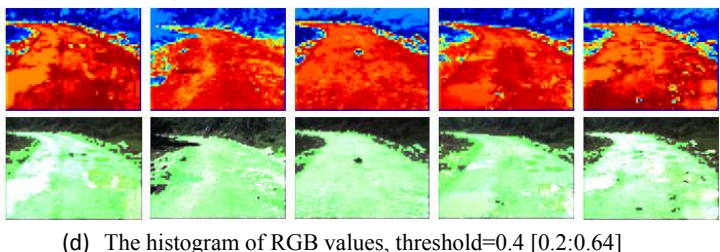

(d) The histogram of RGB values, threshold $=0.4$ [0.2:0.64]
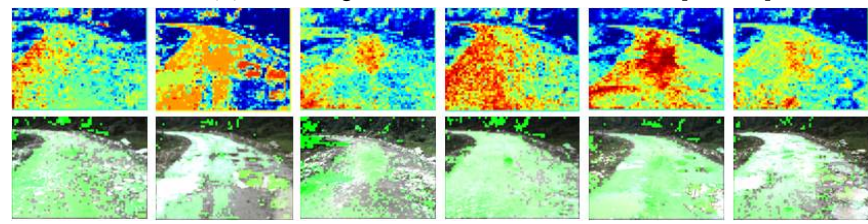

(e) The histogram of difference RGB value, threshold $=0$ [-0.5:0.5]
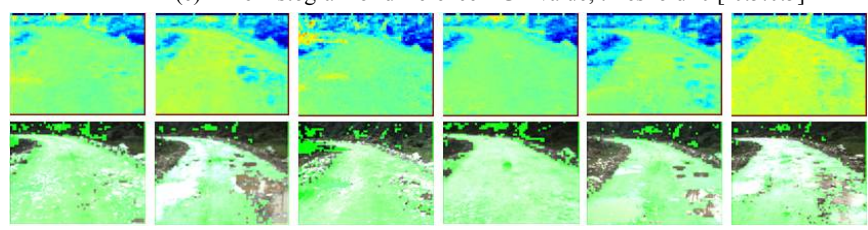

(f) The advanced RGB values, threshold $=-0.35[-0.5: 0]$

Fig. 4. The results of comparing experiments. (a) is the benchmark labeled by human. The upper row in $(b, c, d, e, f)$ are the deep map of margin distance generated by SVM according different kinds of feature descriptors. The lower row in (b,c,d,e,f) are the result of region detection when a fixed threshold is chosen. Different ranges are marked besides of the fixed threshold.

results show that the algorithm based on the average of RGB values and the histogram of RGB values have a better region division than other feature descriptors.

A series of different unstructured road scenes were tested. The ROC (receiver operating characteristic) curve is used to show the effect of these feature descriptors. ROC reflects the true positive rate (hitting rate) against the false positive rate (false alarm rate) while shifting the threshold in a fixed range ( these ranges are marked besides corresponding to each threshold in Fig.4). The area under the ROC curve is called ROC score which reflects the performance of an algorithm. In this experiment, the false positive rate and the true positive rate were computed as follows: in each scene, the number of false positive pixels and the number of true positive pixels were computed under the ground truth at each threshold value. 
Under the same threshold, the numbers of false positive pixels in all different scenes were added together, and the numbers of true positive pixels in all different scenes were added together, too. More than 1000 different images (including different scenarios, road widths, and types) have been computed, the result of ROC curve is shown in Fig.5. In Fig.5, it is very obviously that these ROC curves corresponding to the feature of the average of RGB values (blue) and the histogram of RGB values (yellow) are better than others (black, green and red).

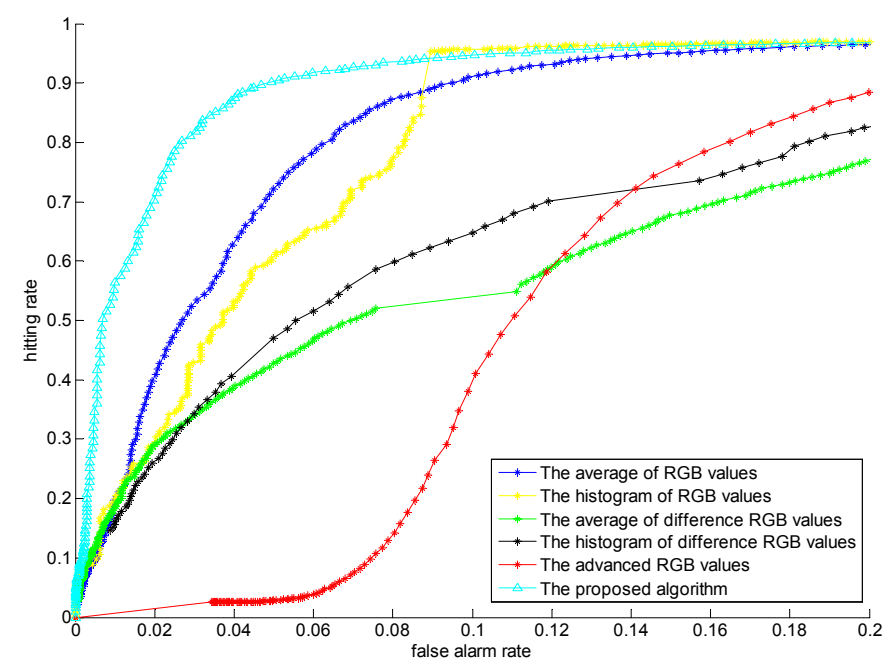

Fig. 5. The ROC curve of the road detection results by different features.

After analyzing kinds of different feature descriptors, we combine two of the most important features, the histogram of RGB values and the average of RGB values, to improve the effect of unstructured road detection. On the same condition, the histogram of RGB values (the vector size is 24) and the average of RGB values (the vector size is 30 ) are combined into a new feature vector (the vector size is 54). Fig.6 and the ROC curve (cyan) in Fig.5 show that the new hybrid-features is more effective than these algorithms based on single feature descriptor.

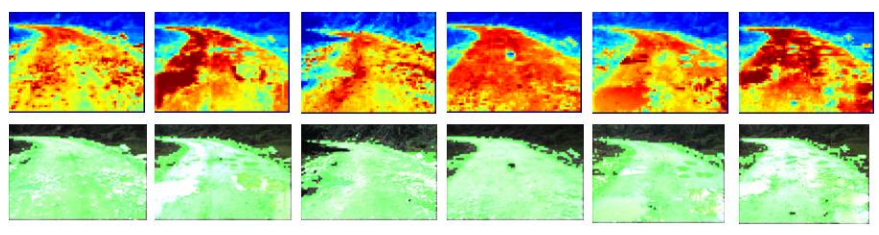

Fig. 6. The experiment result based on hybrid features, threshold $=100$ [60:300].

\section{CONCLUSION}

Unstructured road detection is a challenging problem due to its unstructured nature, variable weather conditions and lighting conditions. Feature descriptor is important for unstructured road detection. In this paper, SVM technology is used to analyze the importance of these common feature descriptors during the road detection process. A series of features are analyzed to show their ability. The experiments show that the analysis of importance to these feature descriptors are consistent to the real road detection. Then, a novel algorithm based on hybrid-feature which are thought important to road detection is presented. The ROC curve shows that the proposed hybrid-feature algorithm is more effectiveness than based on single feature descriptor.

Though only five common feature descriptors are analyzed in this paper, it is the same to analyze other feature descriptor by using the method proposed in this paper.

\section{ACKNOWLEDGE}

This work was supported in part by the National Natural Science Foundation of China under Grant 90820302.

\section{REFERENCES}

[1] J. Wang, Z. Ji, and Y. Su, "Unstructured road detection using hybrid features," in Machine Learning and Cybernetics, 2009 International Conference on, vol. 1. IEEE, 2009, pp. 482-486.

[2] M. Sotelo, F. Rodriguez, L. Magdalena, L. Bergasa, and L. Boquete, "A color vision-based lane tracking system for autonomous driving on unmarked roads," Autonomous Robots, vol. 16, no. 1, pp. 95-116, 2004.

[3] C. Tan, T. Hong, T. Chang, and M. Shneier, "Color model-based realtime learning for road following," in Intelligent Transportation Systems Conference, 2006. ITSC'06. IEEE. IEEE, 2006, pp. 939-944.

[4] Y. Huang and Y. Pan, "Fast algorithm for structured and unstructured road detection," in Image and Signal Processing, 2009. CISP'09. 2nd International Congress on. IEEE, 2009, pp. 1-5.

[5] Q. Gao, Q. Luo, and S. Moli, "Rough set based unstructured road detection through feature learning," in Automation and Logistics, 2007 IEEE International Conference on. IEEE, 2007, pp. 101-106.

[6] M. Foedisch and A. Takeuchi, "Adaptive real-time road detection using neural networks," in Intelligent Transportation Systems, 2004. Proceedings. The 7th International IEEE Conference on. Ieee, 2004, pp. 167172.

[7] Q. Chen and H. Wang, "A real-time lane detection algorithm based on a hyperbola-pair model," in Intelligent Vehicles Symposium, 2006 IEEE. IEEE, 2006, pp. 510-515.

[8] Y. Alon, A. Ferencz, and A. Shashua, "Off-road path following using region classification and geometric projection constraints," in Computer Vision and Pattern Recognition, 2006 IEEE Computer Society Conference on, vol. 1. IEEE, 2006, pp. 689-696.

[9] P. Jeong and S. Nedevschi, "Efficient and robust classification method using combined feature vector for lane detection," Circuits and Systems for Video Technology, IEEE Transactions on, vol. 15, no. 4, pp. 528-537, 2005.

[10] Y. Yim and S. Oh, "Three-feature based automatic lane detection algorithm (tfalda) for autonomous driving," Intelligent Transportation Systems, IEEE Transactions on, vol. 4, no. 4, pp. 219-225, 2003.

[11] J. Alvarez, T. Gevers, and A. Lopez, "3d scene priors for road detection," in Computer Vision and Pattern Recognition (CVPR), 2010 IEEE Conference on. IEEE, 2010, pp. 57-64.

[12] N. Dalal, "Finding people in images and videos," Ph.D. dissertation, Institut National Polytechnique de Grenoble-INPG, 2006. 